\title{
Chern-Simons Origin of Superstring Integrability
}

\author{
Kevin Costello ${ }^{1, *}$ and Bogdan Stefański, Jr. $\oplus^{2,1, \dagger}$ \\ ${ }^{1}$ Perimeter Institute, 31 Caroline Street North, Waterloo, Ontario N2L 2Y5, Canada \\ ${ }^{2}$ Centre for Mathematical Science, City, University of London, Northampton Square, London EC1V OHB, United Kingdom
}

(Received 21 May 2020; revised 4 August 2020; accepted 26 August 2020; published 18 September 2020)

\begin{abstract}
We derive the $\mathrm{AdS}_{5} \times \mathrm{S}^{5}$ Green-Schwarz superstring from four-dimensional Beltrami-Chern-Simons theory reduced on a manifold with singular boundary conditions. In this construction, the Lax connection and spectral parameter of the integrable superstring have a simple geometric origin in four dimensions as gauge connection and reduction coordinate. $\kappa$ symmetry arises as a certain class of singular gauge transformations, while the world-sheet metric comes from complex-structure-changing Beltrami differentials. Our approach offers the possibility of investigating integrable holography using traditional field theory methods.
\end{abstract}

DOI: 10.1103/PhysRevLett.125.121602

Introduction.-Integrability is an invaluable exact tool for AdS/CFT holography [1] which has provided significant evidence in favor of many of the dualities conjectured by Maldacena [2,3]. Signs of integrability were noticed early on in both gauge and string theory. At weak 't Hooft coupling the dilatation operator corresponds to an integrable spin-chain Hamiltonian [4-6], while at strong coupling superstring equations of motion are equivalent to the flatness of an auxiliary Lax connection $[7,8]$. Yet the origin of the integrable structure underlying these theories remains obscure.

In this Letter, we will attempt to demystify the appearance of integrability in holographic superstring theory by showing how Metsaev-Tseytlin (MT) $\kappa$-symmetric string actions $[9,10]$ can be obtained from four-dimensional Chern-Simons $\left(\mathrm{CS}_{4}\right)$ gauge theory with suitably chosen boundary conditions. Recently, a new approach to integrability based on $\mathrm{CS}_{4}$ theory has been proposed in [11-13]. In this approach, reducing $\mathrm{CS}_{4}$ theory to two dimensions in the presence of defects [14] gives rise to many integrable two-dimensional field theories such as the Gross-Neveu and Wess-Zumino-Witten models, as well as the purespinor sigma model on $\mathrm{AdS}_{5} \times \mathrm{S}^{5}$ [15].

We begin by obtaining the MT sigma model from a reduction of $\mathrm{CS}_{4}$ theory generalizing [14]. As in that work, the mysterious Lax connection and spectral parameter of the MT sigma model have a prosaic interpretation in terms of the gauge potential and direction of reduction in the $\mathrm{CS}_{4}$ theory [16]. The absence of fermion kinetic terms makes

Published by the American Physical Society under the terms of the Creative Commons Attribution 4.0 International license. Further distribution of this work must maintain attribution to the author(s) and the published article's title, journal citation, and DOI. Funded by SCOAP ${ }^{3}$. the MT sigma model pathological [17]. Nonetheless, coupling it to a world-sheet metric leads to a consistent string theory [18] with target-space supersymmetry [19] because the action has $\kappa$ symmetry [20].

We couple $\mathrm{CS}_{4}$ theory to complex-structure-changing Beltrami differentials. In the presence of singular boundary conditions, these couplings cannot be removed by field redefinitions and reducing this Beltrami-Chern-Simons (BCS) theory to two dimensions gives the MT string theory. We show that BCS theory has $\kappa$ symmetry, with $\kappa$ transformations implemented by certain singular gauge transformations combined with an action on the Beltrami differentials which has compact support near the singularities. The resulting theory is essentially a conventional gauge theory of Chern-Simons type coupled to extra matter. On general grounds its observables will be Wilson lines whose interactions encode the $R$ matrix of AdS/CFT. These can be computed using Feynman graphs with propagators and vertices derived in a conventional way from the action, taking into account the slightly unusual boundary conditions. As a result, our construction opens up the possibility of investigating integrability in string theory and holography through conventional and rigorous quantum field theory methods, albeit with singular boundary conditions.

MT sigma model from $\mathrm{CS}_{4}$. - Consider the $\mathrm{CS}_{4}$ action on $\mathcal{V}=\Sigma \times C$

$$
S_{\mathrm{CS}_{4}}=\frac{1}{h} \int_{\mathcal{V}} \omega \wedge L_{\mathrm{CS}}(A)
$$

where $L_{\mathrm{CS}}$ is the CS Lagrangian (23), $\Sigma=\mathbb{R}^{2}, C=\mathbb{C P}^{1}$ and the holomorphic one-form $\omega$ has $n$ second-order poles, and $2 n-2$ first-order zeros

$$
\omega=\frac{\prod\left(z-q_{k}\right) \prod\left(z-\tilde{q}_{k}\right)}{\prod\left(z-p_{i}\right)^{2}} d z .
$$


The gauge group of main interest in this Letter is $G=\mathfrak{p} \mathfrak{i t}(2,2 \mid 4)$ whose dual Coxeter number is zero. As a result, the theory is framing-anomaly free and $h$ is not quantized. As reviewed in [18], $\mathfrak{p} \mathfrak{g} \mathfrak{\mathfrak { t }}(2,2 \mid 4)$ has a $\mathbb{Z}_{4}$ automorphism and we denote its $i^{m}$ eigenspace by $\mathfrak{p} \mathfrak{g} \mathfrak{t}(2,2 \mid 4)^{(m)} \quad(m=0, \ldots, 3)$ and recall that $\mathfrak{p} \mathfrak{g} \mathfrak{t}(2,2 \mid 4)^{(0)}=\mathfrak{g} \mathfrak{o}(4,1) \times \mathfrak{S} \mathfrak{o}(5)$. As in [14], for a welldefined action we require $A_{w}, A_{\bar{w}}$ to have first-order zeros at $z=p_{i}$ and poles at

$$
\left.A_{w}\right|_{z=q_{k}} \sim \frac{1}{z-q_{k}},\left.\quad A_{\bar{w}}\right|_{z=\tilde{q}_{k}} \sim \frac{1}{z-\tilde{q}_{k}} .
$$

Solving the equations of motion and boundary conditions gives $A=\hat{A}+A^{\prime}$, where $\hat{A}=\hat{\sigma}^{-1} d \hat{\sigma}, A^{\prime}=\hat{\sigma}^{-1} \mathcal{L} \hat{\sigma}$, with $\mathcal{L}_{\bar{z}}=0$ and

$$
\begin{aligned}
\mathcal{L}_{w} & =\frac{\prod\left(z-p_{i}\right)}{\prod\left(z-q_{k}\right)} \sum_{j} \frac{1}{p_{j}-z} \frac{\prod\left(p_{j}-q_{k}\right)}{\prod_{i \neq j}\left(p_{j}-p_{i}\right)} \partial_{w} \sigma_{j} \sigma_{j}^{-1}, \\
\mathcal{L}_{\bar{w}} & =\frac{\prod\left(z-p_{i}\right)}{\prod\left(z-\tilde{q}_{k}\right)} \sum_{j} \frac{1}{p_{j}-z} \frac{\prod\left(p_{j}-\tilde{q}_{k}\right)}{\prod_{i \neq j}\left(p_{j}-p_{i}\right)} \partial_{\bar{w}} \sigma_{j} \sigma_{j}^{-1} .
\end{aligned}
$$

The notation used above follows [14]. In particular, up to gauge transformations, $A_{\bar{z}}$ defines a map $\sigma: \mathbb{R}^{2} \rightarrow G^{n}$, which extends to $\hat{\sigma}: \mathcal{V} \rightarrow G^{n}$, where near $z=p_{i}, \hat{\sigma} \sim \sigma_{i}$, with $\sigma_{i}: \mathbb{R}^{2} \rightarrow G$. In [14] constant gauge transformations were used to set $\sigma_{n}=$ Id. Inserting $A$ back into $S_{\mathrm{CS}_{4}}$ localizes the action on the boundary and gives a large family of integrable sigma models, with left-acting $G^{n}$ symmetry and Lax connection $\mathcal{L}$ [14], which were also found in [21] without reference to $\mathrm{CS}_{4}$ theory.

We now set $p_{j}=\exp (2 \pi i j / n)$ and, in contradistinction to [14], take the limit

$$
\begin{aligned}
& q_{1}=\ldots=q_{n-m}=\tilde{q}_{n-m+1}=\ldots=\tilde{q}_{n-1} \rightarrow 0, \\
& \tilde{q}_{1}=\ldots=\tilde{q}_{n-m}=q_{n-m+1}=\ldots=q_{n-1} \rightarrow \infty,
\end{aligned}
$$

with $1<m<n$. Upon rescaling $h \rightarrow h\left(-\tilde{q}_{1}\right)^{n-1}$, the $m=2$ action is

$$
\begin{aligned}
S^{n, m=2}= & \frac{k}{4 \pi n} \int_{\Sigma} \sum_{i} J_{i, w} J_{i, \bar{w}}-\sum_{i, j} \alpha_{i j} J_{i, \bar{w}} J_{j, w} \\
& -\frac{k}{8 \pi n^{2}} \int_{\Sigma} \sum_{i \neq j} \frac{p_{i}^{3}+p_{j}^{3}}{p_{i} p_{j}\left(p_{i}-p_{j}\right)} J_{i, \bar{w}} J_{j, w} \\
& +\frac{k}{12 \pi n^{2}} \int_{\Sigma \times \mathbb{R}^{+}} f^{a b c} X_{i, a} X_{i, b} X_{i, c},
\end{aligned}
$$

where $J_{i} \equiv d \sigma_{i} \sigma_{i}^{-1}, X_{i} \equiv \sigma_{i}^{-1} d \sigma_{i} f^{a b c}$ are the gauge-group structure constants, $\mathbb{R}^{+}$is the $z$-plane radial direction, $\alpha_{i j} \equiv\left(1+p_{i} / p_{j}+p_{j} / p_{i}\right) / n$ and $k=8 \pi i / h$ [22].

These models have a $\mathbb{Z}_{n}$ symmetry $\rho$, which permutes the $n$ copies of $G$, together with acting by the $\mathbb{Z}_{n}$ automorphism on each copy, and multiplies $z$ by an $n$th root of unity. This fixes a subgroup $\rho\left(G^{(0)}\right)=G^{(0)} \subset G$, which for $G=\mathfrak{p} \mathfrak{g} \mathfrak{t}(2,2 \mid 4)$ is $G^{(0)}=\mathfrak{g} \mathfrak{o}(4,1) \times \mathfrak{g} \mathfrak{o}(5)$. Gauging the $\mathbb{Z}_{n}$ action leads to novel integrable models on generalized symmetric spaces $G / G^{(0)}$ whose equations of motion are equivalent to the flatness condition of the Lax connection

$$
\begin{aligned}
& \mathcal{L}_{w}^{n, m}(z)=\sum_{k=0}^{m} z^{k} \boldsymbol{J}_{w}^{(k)}+\sum_{k=m+1}^{n-1} z^{k-n} \boldsymbol{J}_{w}^{(k)} \\
& \mathcal{L}_{\bar{w}}^{n, m}(z)=\sum_{k=0}^{n-m-1} z^{k} \boldsymbol{J}_{\bar{w}}^{(k)}+\sum_{k=n-m}^{n-1} z^{k-n} \boldsymbol{J}_{\bar{w}}^{(k)},
\end{aligned}
$$

where $J_{a} \equiv J_{1, a}, \quad J_{i, a}=\rho^{i-1}\left(J_{a}\right)$, and $J^{(k)}$ is the $k$ th $\mathbb{Z}_{n}$ eigenspace. In particular, for $m=2, n=4$ we obtain the MT $\sigma$ model $[9,10,23]$ with target-space $\mathfrak{p} \mathfrak{B} \mathfrak{u}(2,2 \mid 4) /[\mathfrak{g} \mathfrak{v}(4,1) \times \mathfrak{S} \mathfrak{o}(5)]$

$$
S^{n=4, m=2}=\frac{k}{4 \pi} \int_{\Sigma} J_{w}^{(2)} J_{\bar{w}}^{(2)}-J_{w}^{(1)} J_{\bar{w}}^{(3)}+J_{w}^{(3)} J_{\bar{w}}^{(1)} .
$$

Metric and Virasoro constraints.-The $S^{n, m}$ models, unlike their pure-spinor counterparts [14], do not have second-order kinetic terms for fermions, since the boundary conditions imposed in $\mathrm{CS}_{4}$ to obtain them are not elliptic. This apparent disadvantage is in fact a boon: for judicious choices of $G, S^{4,2}$ has $\kappa$ symmetry when coupled to the world-sheet metric.

In our derivation of $S^{4,2}$ from $\mathrm{CS}_{4}$ theory it is not immediately clear how a world-sheet metric might arise. After all, $\mathrm{CS}_{4}$ theory does not depend on the fourdimensional metric. More precisely, this is only true for manifolds without boundary or for everywhere regular field configurations. On the other hand, allowing singularities in the gauge field like those in Eq. (3) means that the $\mathrm{CS}_{4}$ theory may no longer be invariant under general-coordinate transformations on the boundary or in regions near such singularities. If we separate the singular part of the gauge connection

$$
A_{w} \equiv A_{w}^{(p)}+A_{w}^{(\mathrm{reg})}, \quad A_{\bar{w}} \equiv A_{\bar{w}}^{(p)}+A_{\bar{w}}^{(\mathrm{reg})},
$$

$S_{\mathrm{CS}_{4}}$ is not general-coordinate invariant

$$
\delta_{v} S_{\mathrm{CS}_{4}} \sim-\oint_{z=0, \infty} \omega_{\wedge}\left(A_{w}^{(p)} d v^{w}+A_{\bar{w}}^{(p)} d v^{\bar{w}}\right)_{\wedge} A .
$$

We used Green's theorem to rewrite $\delta_{v} S_{\mathrm{CS}_{4}}$ as a contour integral around $z=0, \infty$, where $A_{w}$ and $A_{\bar{w}}$ have poles. For $S^{4,2}$ this integral reduces to

$$
-\int_{\Sigma}\left(A_{w}^{(2)} A_{w}^{(2)} d w_{\wedge} d v^{w}+A_{\bar{w}}^{(2)} A_{\bar{w}}^{(2)} d \bar{w}_{\wedge} d v^{\bar{w}}\right)
$$


Since this is nonzero, it appears that with our boundary conditions the $\mathrm{CS}_{4}$ theory is no longer invariant under general-coordinate transformations.

The lack of diffeomorphism invariance (12) suggests we need to introduce a new field to restore it. We do this by varying the complex structure with the new field corresponding to the Beltrami differential $\beta$. Under such a variation, the de Rham differential $d$, which is the $\mathrm{CS}_{4}$ theory's BRST operator, changes to

$$
d \rightarrow d+d \bar{w} \mathcal{L}_{\beta_{\bar{w}} \partial_{w}}+d \bar{z} \mathcal{L}_{\beta_{\bar{z}} \partial_{w}},
$$

where $\mathcal{L}$ is the Lie derivative. Since the Cartan homotopy formula gives the Lie derivative as

$$
\mathcal{L}_{V}=\left[d, \iota_{V}\right],
$$

where $l_{V}$ is the inner multiplication (contraction) by $V$, we are adding a BRST-exact term to the action, which should have no effect in the bulk. The $\beta_{\bar{w}}$-dependent part of the action is

$$
S_{\beta_{\bar{w}}} \equiv \frac{2}{h} \int_{\mathcal{V}} \omega_{z} \beta_{\bar{w}} A_{w}\left(\partial_{\bar{z}} A_{w}-\partial_{w} A_{\bar{z}}\right),
$$

with a similar term for $S_{\beta_{\bar{z}}}$. The combined BeltramiChern-Simons action

$$
S_{\mathrm{BCS}} \equiv S_{C S_{4}}+S_{\beta_{\bar{w}}}+S_{\beta_{\bar{z}}}
$$

is invariant, up to a $w$ derivative, under a new gauge invariance

$$
A \rightarrow A+\mathcal{L}_{V} A, \quad \beta \rightarrow \beta+\mathcal{L}_{V} \beta,
$$

with gauge parameter $V \equiv v \partial_{w}$ for an arbitrary function $v$. This invariance can be used to gauge away one of the components of $\beta$, for example $\beta_{\bar{z}} \rightarrow 0$. Working in this gauge, redefining $A_{\bar{w}}$

$$
A_{\bar{w}} \rightarrow A_{\bar{w}}-\beta_{\bar{w}} A_{w},
$$

one recovers the original $\mathrm{CS}_{4}$ action

$$
S_{\mathrm{CS}_{4}}+S_{\beta_{\bar{w}}} \rightarrow S_{\mathrm{CS}_{4}} \text {. }
$$

This is to be expected, since on a manifold with no boundary the $\mathrm{CS}_{4}$ theory is metric independent and we are adding a BRST-exact Beltrami term to it. This should leave the theory unmodified, up to field redefinitions. However, in the presence of a boundary the field redefinition (18) might not be compatible with the boundary conditions. Indeed, $A_{\bar{w}}$ and $A_{w}$ have poles of different order at $z=0, \infty$, while $A_{\bar{z}}$ is regular. Since $\beta$ should be regular on the boundary, this means that we cannot eliminate the Beltrami couplings on the boundary using field redefinitions (18). With boundary conditions (6) and (3), the $\beta$-dependent part of the action reduces to a boundary contribution at $z=0$

$$
S_{\beta}=\frac{\delta_{z=0}}{h} \int_{\Sigma} 2 \beta_{\bar{w}} A_{w}^{(2)} A_{w}^{(2)} .
$$

This coupling restores diffeomorphism invariance at $z=0$ and varying the action with respect to $\beta_{\bar{w}}$ leads to the Virasoro constraint

$$
A_{w}^{(2)} A_{w}^{(2)}=0
$$

We can introduce a similar modification to Eq. (13) along $\partial_{\bar{w}}$ and show that it is trivial up to a field redefinition away from $z=\infty$, leading to a boundary action

$$
S_{\tilde{\beta}_{w}}=\frac{\delta_{z=\infty}}{h} \int_{\Sigma} 2 \tilde{\beta}_{w} A_{\bar{w}}^{(2)} A_{\bar{w}}^{(2)},
$$

which restores diffeomorphism invariance at $z=\infty$.

In the Polyakov action, the world-sheet metric $g$ is taken up to Weyl transformations. If we analytically continue this action to allow $g$ to be a metric with complex coefficients, then the data of $g$, up to Weyl transformations, is equivalent to the data of a holomorphic Beltrami differential $\beta$ and an antiholomorphic Beltrami differential $\bar{\beta}$ [24]. The reality condition corresponding to asking that $g$ be a metric with real coefficients is that we ask $\beta$ and $\bar{\beta}$ to be complex conjugate. For a discussion of such a factorization of the Polyakov action see [25].

Similarly, in the Polyakov action, the gauge symmetries (after gauging away Weyl transformations) are world-sheet diffeomorphisms. Infinitesimally these are sections of the tangent bundle $T \Sigma$. If we analytically continue the Polyakov action, allowing the infinitesimal world-sheet diffeomorphisms to be complex, we find the gauge transformations we used for the Beltrami differential fields. Indeed, $T \Sigma \bigotimes_{\mathbb{R}} \mathbb{C}$ decomposes as $T^{1,0} \Sigma$, which gives the gauge transformations for $\beta$, and $T^{0,1} \Sigma$, giving the gauge transformations for $\bar{\beta}$.

Gauge invariance in BCS theory.-Before proceeding to discuss $\kappa$ symmetry in BCS theory, we briefly review how to modify gauge variations in the presence of a Beltrami deformation $\beta_{w}$ in order for $S_{\mathrm{BCS}}$ to be gauge invariant. In components the CS Lagrangian is

$\frac{1}{2} L_{\mathrm{CS}}=A_{\bar{w}}\left(\partial_{\bar{z}} A_{w}-\partial_{w} A_{\bar{z}}\right)+A_{\bar{z}} \partial_{w} A_{\bar{w}}-A_{\bar{w}}\left[A_{w}, A_{\bar{z}}\right]$,

where as in the rest of the paper the trace is implicit. The $\beta_{w} \equiv \beta$ part of the Beltrami-deformed action is

$$
S_{\beta}=-\frac{1}{h} \int_{\mathcal{V}} \omega_{z}\left(\partial_{\bar{z}} \beta_{\bar{w}} A_{w}^{2}+2 \beta_{\bar{w}} A_{w} \partial_{w} A_{\bar{z}}\right) .
$$


$S_{\mathrm{CS}_{4}}$ is invariant under gauge transformations

$$
\delta_{\chi} A_{\mu}=\partial_{\mu} \chi+\left[A_{\mu}, \chi\right],
$$

but $S_{\beta}$ is not

$$
\delta_{\chi} S_{\beta}=2 \int_{\mathcal{V}} \omega \beta_{\bar{w}}\left(\partial_{\bar{z}} A_{w}-\partial_{w} A_{\bar{z}}-\left[A_{w}, A_{\bar{z}}\right]\right) \partial_{w} \chi .
$$

To cancel this we modify the gauge variation of $A_{\bar{w}}$ in accordance with Eq. (13) to

$$
\delta_{\chi, \beta} A_{\bar{w}}=\partial_{\bar{w}} \chi+\left[A_{\bar{w}}, \chi\right]-\beta_{\bar{w}} \partial_{w} \chi,
$$

while leaving the gauge variation of the other components of $A$ unchanged. Since $S_{\beta}$ does not depend on $A_{\bar{w}}$ its variation (26) is unchanged, while the gauge variation of $S_{\mathrm{CS}_{4}}$ becomes

$$
\delta_{\chi, \beta} S_{\mathrm{CS}_{4}}=-\delta_{\chi} S_{\beta},
$$

making $S_{\mathrm{BCS}}$ gauge invariant.

$\kappa$ symmetry.-We now show that the action $S_{\mathrm{BCS}}$ is invariant under certain singular $G$-gauge transformations, which reduce to $\kappa$ symmetry in the $\sigma$ model. To this end, consider gauge variations

$$
\delta_{\xi} A=d \xi+[A, \xi]
$$

which have a simple pole near $z=0$

$$
\xi \sim \frac{1}{z} \xi^{(3)}+\cdots .
$$

Since the generator of the $\mathbb{Z}_{4}$ automorphism multiplies $z$ by $-i$, we immediately see that the singular gauge variation $\xi^{(3)}$ must be in the $i^{3}$ eigenspace of $\mathbb{Z}_{4}$ and hence is fermionic, as expected of a $\kappa$ variation. The variation of $S_{\mathrm{CS}_{4}}$ is

$$
\delta_{\xi} S_{\mathrm{CS}_{4}}=\frac{1}{h} \int_{\mathcal{V}} \omega\left(A_{\bar{w}} d_{\bar{z}}\left[A_{w}, \xi\right]-A_{w} d_{\bar{z}}\left[A_{\bar{w}}, \xi\right]\right) .
$$

Near $z=0$ the gauge fields have an expansion

$$
A_{\bar{w}} \sim \frac{A_{\bar{w}}^{(3)}}{z}+\cdots, \quad A_{w} \sim \frac{A_{w}^{(2)}}{z^{2}}+\frac{A_{w}^{(3)}}{z}+\cdots .
$$

Inserting these into (31) we get [26]

$$
\delta_{\xi} S_{\mathrm{CS}_{4}}=\frac{\delta_{z=0}}{h} \int_{\Sigma}\left[A_{\bar{w}}^{(3)}, A_{w}^{(2)}\right] \xi^{(3)} .
$$

Analogously, for gauge variations with a simple pole at $z=\infty$, we have $\xi \sim z \xi^{(1)}+\cdots$, and the $\mathrm{CS}_{4}$ action changes by

$$
\delta_{\tilde{\xi}} S_{\mathrm{CS}_{4}}=\frac{\delta_{z=\infty}}{h} \int_{\Sigma}\left[A_{w}^{(1)}, A_{\bar{w}}^{(2)}\right] \tilde{\xi}^{(1)} .
$$

$\kappa$ transformations can be obtained from the singular gauge transformations by requiring [18]

$$
\begin{aligned}
& \tilde{\xi}^{(1)} \equiv A_{\bar{w}}^{(2)} \kappa_{w}^{(1)}+\kappa_{w}^{(1)} A_{\bar{w}}^{(2)}, \\
& \xi^{(3)} \equiv A_{w}^{(2)} \kappa_{\bar{w}}^{(3)}+\kappa_{\bar{w}}^{(3)} A_{w}^{(2)},
\end{aligned}
$$

where the $\kappa$ are the independent (local) parameters of $\kappa$ transformations. Notice that the above expression involves selecting a particular (matrix) representation for the gauge group, and using matrix multiplication in that representation. In judiciously chosen cases, there are certain famous Fierz identities [18,27,28] [see Eq. (1.80) of [18] ] that can be used to reexpress the $\kappa$ variation of $S_{\mathrm{CS}_{4}}$ as

$$
\begin{aligned}
\delta_{\kappa} S_{\mathrm{CS}_{4}}= & -\frac{\delta_{z=0}}{2 h} \int_{\Sigma} \operatorname{tr}\left(A_{w}^{(2)} A_{w}^{(2)}\right) \operatorname{tr}\left(\Upsilon\left[\kappa_{\bar{w}}^{(3)}, A_{\bar{w}}^{(3)}\right]\right) \\
& -\frac{\delta_{z=\infty}}{2 h} \int_{\Sigma} \operatorname{tr}\left(A_{\bar{w}}^{(2)} A_{\bar{w}}^{(2)}\right) \operatorname{tr}\left(\Upsilon\left[\kappa_{w}^{(1)}, A_{w}^{(1)}\right]\right) .
\end{aligned}
$$

Above, $\Upsilon$ is a suitable constant matrix which for $\mathfrak{p} \mathfrak{g} \mathfrak{t}(2,2 \mid 4)$ is $\operatorname{diag}\left(1_{4},-1_{4}\right)$. The lack of gauge invariance under (30) can be compensated by varying the Beltrami operators under $\kappa$ transformations. Working in the gauge $\beta_{\bar{z}}=0$, we demand

$$
\delta_{\kappa} \beta_{\bar{w}}=\frac{\delta_{|z| \leq \varepsilon}}{2} \operatorname{tr}\left(\Upsilon\left[\kappa_{\bar{w}}^{(3)}, A_{\bar{w}}^{(3)}\right]\right),
$$

where $\delta_{|z| \leq \varepsilon}$ has support in an $\varepsilon$ neighborhood of $z=0$ only. Now, $S_{\beta_{\bar{w}}}$ is no longer gauge invariant near $z=0$. Expanding as in Eq. (32), we find

$$
\begin{aligned}
\delta_{\kappa} S_{\beta} & =\frac{1}{2 h} \int_{\mathcal{V}_{4}} \frac{\partial_{\bar{z}} \delta_{|z| \leq \varepsilon}}{z} \operatorname{tr}\left(\Upsilon\left[\kappa_{\bar{w}}^{(3)}, A_{\bar{w}}^{(3)}\right]\right) \operatorname{tr}\left(A_{w}^{(2)} A_{w}^{(2)}\right) \\
& =\frac{1}{2 h} \oint_{z=0} \frac{d z}{z} \int_{\Sigma} \operatorname{tr}\left(\Upsilon\left[\kappa_{\bar{w}}^{(3)}, A_{\bar{w}}^{(3)}\right]\right) \operatorname{tr}\left(A_{w}^{(2)} A_{w}^{(2)}\right)
\end{aligned}
$$

using the identity $\partial_{\bar{z}} \delta_{|z| \leq \varepsilon}=\delta_{|z|=\varepsilon}$. This cancels the gauge noninvariance of $S_{\mathrm{CS}_{4}}$ at $z=0$ in Eq. (36). The $z=\infty$ term can be analogously canceled by a $\tilde{\beta}_{w}$ variation. The separate cancelations at $z=0, \infty$, which on the world sheet correspond to self-dual- and anti-self-dual-vector representations or after Wick rotation holomorphic and antiholomorphic ones, provide a novel separation of the two sectors in four dimensions.

Conclusions.-In this Letter, we have introduced BCS theory and showed that, upon imposing suitable singular boundary conditions, it reduces to the MT superstring. The Beltrami fields and boundary gauge connection of BCS theory map to the world-sheet metric and sigma-model 
fields, respectively. The Lax connection and spectral parameter appear somewhat mysteriously in string theory, but from the four-dimensional BCS point of view they are simply the gauge connection and holomorphic coordinate of the reduction. In BCS theory $\kappa$ symmetry corresponds to a certain class of singular gauge transformations and $\kappa$ invariance holds for gauge groups for which a suitable hypercharge matrix $\Upsilon$ exists. This includes $G=$ $\mathfrak{p} \mathfrak{B} \mathfrak{t}(2,2 \mid 4)$ and its plane-wave [29] and flat-space [10] limits. We will investigate the properties of these backgrounds from the four-dimensional point of view more fully in a forthcoming paper [30].

There are other well-known integrable superstring backgrounds with a Lax connection [31-37]. In these cases, the MT coset action often needs to be supplemented by extra fermionic degrees of freedom [38] to obtain an action equivalent to the conventional $\kappa$-symmetric superstring actions $[39,40]$ and it would be interesting to see how to extend these to BCS theory. Some of these backgrounds have target-space moduli and understanding how these appear in BCS theory could provide new insights into moduli spaces.

It would be interesting to perform a Batalin-Vilkovisky quantization of BCS theory. This introduces a tower of extra fields including conventional $b-c$ ghosts of string theory. If the $\mathbb{R}^{2}$ with coordinates $w, \bar{w}$ is replaced by a Riemann surface $\Sigma$, then the formalism we have described includes the integral over the moduli of the world-sheet $\Sigma$. Indeed, the Beltrami differential $\beta$ on $\Sigma$ has zero modes which live in the Dolbeault cohomology group $H^{1}(\Sigma, T \Sigma)$, which is of (complex) dimension $3 g-3$ for $g>1$ [41]. The antiholomorphic Beltrami differential has zero modes which live in the complex conjugate of this space. Together, the manifold of zero modes is the product of the moduli space with its complex conjugate: $\mathcal{M}_{g} \times \overline{\mathcal{M}}_{g}$. As we are doing an analytically continued path integral, we need to choose an integration contour. It is natural to choose the contour to be the locus where the holomorphic and antiholomorphic Beltrami differential are complex conjugate, leading to an integral over one copy of the moduli space $\mathcal{M}_{g}$. We will return to a detailed discussion of this and its relation to the Polyakov path integral over Riemann surfaces [42] in a future paper [30].

We hope our construction can shed light on the relationship between the pure-spinor and Green-Schwarz formulations of string theory. Quantizing BCS theory should also offer new insights into quantum integrability of holographic string backgrounds and connect with the quantum spectral curve approach $[43,44]$. In particular, we expect that compactifying the string world sheet $\Sigma=\mathbb{R} \times S^{1}$ will lead to diagrams involving the photon propagator "wrapping" the $S^{1}$ direction and the $\mathbb{Q}$ functions should appear as solutions of the Baxter equation involving the transfer matrix of BCS theory.
We would like to thank Masahito Yamazaki for discussions. This research was supported in part by a grant from the Krembil Foundation. K. C. is supported by the NSERC Discovery Grant program and by the Perimeter Institute for Theoretical Physics. Research at Perimeter Institute is supported in part by the Government of Canada through the Department of Innovation, Science and Economic Development Canada and by the Province of Ontario through the Ministry of Colleges and Universities. B.S. acknowledges funding support from an STFC Consolidated Grant Theoretical Physics at City University ST/P000797/1. B. S. is grateful for the hospitality of Perimeter Institute where part of this work was carried out. Research at Perimeter Institute is supported in part by the Government of Canada through the Department of Innovation, Science and Economic Development Canada and by the Province of Ontario through the Ministry of Colleges and Universities.

*kcostello@perimeterinstitute.ca 'Bogdan.Stefanski.1@ city.ac.uk

[1] N. Beisert et al., Lett. Math. Phys. 99, 3 (2012).

[2] J. M. Maldacena, Adv. Theor. Math. Phys. 2, 231 (1998).

[3] For a recent pedagogical review of integrability and its holographic applications, see D. Bombardelli, A. Cagnazzo, R. Frassek, F. Levkovich-Maslyuk, F. Loebbert, S. Negro, I. M. Szécsényi, A. Sfondrini, S. J. van Tongeren, and A. Torrielli, J. Phys. A 49, 320301 (2016).

[4] J. Minahan and K. Zarembo, J. High Energy Phys. 03 (2003) 013.

[5] N. Beisert and M. Staudacher, Nucl. Phys. B670, 439 (2003).

[6] N. Beisert, Nucl. Phys. B682, 487 (2004).

[7] I. Bena, J. Polchinski, and R. Roiban, Phys. Rev. D 69, 046002 (2004).

[8] G. Mandal, N. V. Suryanarayana, and S. R. Wadia, Phys. Lett. B 543, 81 (2002).

[9] R. Metsaev and A. A. Tseytlin, Nucl. Phys. B533, 109 (1998).

[10] M. Henneaux and L. Mezincescu, Phys. Lett. 152B, 340 (1985).

[11] K. Costello, arXiv:1303.2632.

[12] K. Costello, E. Witten, and M. Yamazaki, ICCM Not. 6, 46 (2018).

[13] K. Costello, E. Witten, and M. Yamazaki, ICCM Not. 6, 120 (2018).

[14] K. Costello and M. Yamazaki, arXiv:1908.02289.

[15] N. Berkovits, J. High Energy Phys. 04 (2000) 018.

[16] This construction generalizes to a large class of MT-like integrable models.

[17] At quadratic order in fields, the kinetic terms in the MT sigma-model (9) involve only bosonic fields $J^{(2)}$. This is in counter-distinction to the pure-spinor model which contains additional kinetic terms for fermionic components $J^{(1)}$ and $J^{(3)}$. 
[18] G. Arutyunov and S. Frolov, J. Phys. A 42, 254003 (2009).

[19] M. B. Green and J. H. Schwarz, Phys. Lett. 136B, 367 (1984).

[20] W. Siegel, Phys. Lett. 128B, 397 (1983).

[21] F. Delduc, S. Lacroix, M. Magro, and B. Vicedo, Phys. Rev. Lett. 122, 041601 (2019).

[22] The factor of $i$ is a consequence of a Euclidean world sheet.

[23] N. Berkovits, M. Bershadsky, T. Hauer, S. Zhukov, and B. Zwiebach, Nucl. Phys. B567, 61 (2000).

[24] To see this, recall that a conformal structure on an oriented surface is the same data as a complex structure, or equivalently, an anticomplex structure. If we analytically continue, these two pieces of data need no longer be the same, so that an analytically continued conformal deformation of the Riemann surface $\Sigma$ is equivalent to a deformation of the complex structure $\Sigma$ and of the complex conjugate surface $\bar{\Sigma}$. In more prosaic terms, a deformation of the metric $g$ has three components, one of which can be removed by a Weyl transformation. The other two are, once we complexify, sections of $\left(T^{1,0} \Sigma\right)^{\otimes 2}$ and $\left(T^{0,1} \Sigma\right)^{\otimes 2}$. Using the metric we are deforming to identify $T^{0,1} \Sigma$ with the dual of $T^{1,0} \Sigma$, these two components become the holomorphic and antiholomorphic Beltrami differential.

[25] O. Gwilliam and B. Williams, arXiv:1711.05823.

[26] We use the fact that $\operatorname{tr}\left(g^{(3)} h^{(2)}\right)=0$ for arbitrary elements $g$, $h$ of the Lie algebra.

[27] L. Brink, J. H. Schwarz, and J. Scherk, Nucl. Phys. B121, 77 (1977).
[28] F. Gliozzi, J. Scherk, and D. I. Olive, Nucl. Phys. B122, 253 (1977).

[29] R. R. Metsaev and A. A. Tseytlin, Phys. Rev. D 65, 126004 (2002).

[30] K. Costello and B. Stefański (to be published).

[31] B. Stefański, Jr, Nucl. Phys. B808, 80 (2009).

[32] G. Arutyunov and S. Frolov, J. High Energy Phys. 09 (2008) 129.

[33] A. Babichenko, B. Stefański, Jr., and K. Zarembo, J. High Energy Phys. 03 (2010) 058.

[34] O. O. Sax and B. Stefański, Jr, J. High Energy Phys. 08 (2011) 029.

[35] A. Cagnazzo and K. Zarembo, J. High Energy Phys. 11 (2012) 133; 04 (2013) 003.

[36] D. Sorokin, A. Tseytlin, L. Wulff, and K. Zarembo, J. Phys. A 44, 275401 (2011).

[37] B. Hoare, A. Pittelli, and A. Torrielli, J. High Energy Phys. 11 (2014) 051.

[38] J. Gomis, D. Sorokin, and L. Wulff, J. High Energy Phys. 03 (2009) 015.

[39] M. T. Grisaru, P. S. Howe, L. Mezincescu, B. Nilsson, and P. Townsend, Phys. Lett. 162B, 116 (1985).

[40] M. Duff, P. S. Howe, T. Inami, and K. Stelle, Phys. Lett. B 191, 70 (1987).

[41] For $g=1$ the Beltrami differential has one zero mode.

[42] A. M. Polyakov, Phys. Lett. 103B, 207 (1981).

[43] N. Gromov, V. Kazakov, S. Leurent, and D. Volin, Phys. Rev. Lett. 112, 011602 (2014).

[44] D. Bombardelli, A. Cavagli, D. Fioravanti, N. Gromov, and R. Tateo, J. High Energy Phys. 09 (2017) 140. 